\title{
Estimación de los años de vida potencial perdidos asociados al cierre de las escuelas primarias durante la pandemia de COVID-19
}

Estimated years of potential life lost associated with the closing of elementary schools during the COVID-19 pandemic

\section{Comentado de:}

Christakis DA, et al. JAMA Netw Open. 2020 Nov 2;3(11):e2028786. PMID: $33180132^{1}$

\section{Objetivo}

Estimar los años de vida potencial perdidos (AVPP) asociados a la pandemia de COVID-19 en función de que las escuelas primarias estén cerradas o permanezcan abiertas.

\section{Métodos}

Modelo analítico de decisión para estimar la asociación entre el cierre de escuelas primarias en los EE.UU. y la reducción de los logros educativos, y por otro lado, la asociación entre la reducción de los logros educativos y la esperanza de vida, utilizando fuentes de datos disponibles en forma pública. También se estimó el aumento de la mortalidad directa por COVID-19 y el aumento potencial de la mortalidad que podría haberse producido si la apertura de las escuelas condujera a un aumento de la transmisión de COVID-19.

Los resultados principales se presentaron en AVPP con sus correspondientes intervalos de credibilidad (ICr) del $95 \%$. El concepto de AVPP representa, desde el punto de vista de la sociedad, los años de vida perdidos debido a mortalidad prematura, considerando la expectativa de vida de esa sociedad en ese momento histórico ${ }^{2}$. El ICr, derivado de la estadística Bayesiana, se refiere al intervalo en el cuál existe una cierta probabilidad (en este caso, $95 \%$ ) de que se encuentre el parámetro estimado ${ }^{3}$.

\section{Resultados}

Los niños entre 5 y 11 años de edad perdieron una mediana de 54 días de instrucción (IIC 48 a 62,5), lo que se asoció con una pérdida media de 0,31 ( $\mathrm{ICr} 95 \%, 0,10$ a 0,65$)$ años de logro educativo final para los niños y 0,21 (ICr $95 \% 0,06$ a 0,46) para las niñas. Sumando toda la población, se estima que 5,53 millones (ICr $95 \% 1,88$ a 10,80) de AVPP pueden estar asociados al cierre de escuelas.

Las muertes por COVID-19 durante el mismo periodo representaron 1,50 millones (ICr 95\% 1,23 a 1,85 millones) de AVPP. Si las escuelas hubieran permanecido abiertas, se podrían haber esperado 1,47 millones ( $\mathrm{ICr} 95 \%$ 0,45 a 2,59) de AVPP adicionales.

Al comparar escuelas "abiertasz çerradas", hubo una probabilidad de $98,1 \%$ de que la apertura de las escuelas se hubiera asociado con menor AVPP, y de $53,1 \%$ si se aplicaba una tasa de descuento social del $3 \%$.

\section{Conclusiones}

El análisis estimó como mejor estrategia que las escuelas permanecieran abiertas.

Fuente de financiamiento/Conflicto de interés de los autores: No referidos.

\section{Comentario}

La pandemia generó graves consecuencias para la población, no solamente mortalidad directamente relacionada por el virus, sino también importante morbimortalidad por otros problemas de salud, y enormes disrupciones sociales, pobreza e inequidad $^{4}$.

En Argentina, ante la demora y dificultad en la adopción de medidas más específicas y eficientes para controlar la epidemia, como testear, trazar y aislar, se decidió adoptar una estrategia de aislamiento social obligatorio sostenida en el tiempo. Nuestras autoridades planteaban que con el aislamiento se estaba priorizando evitar muertes por sobre cualquier otra cosa. El problema es que no se dimensionaron los graves efectos adversos de esas medidas autoimpuestas. La relevancia del estudio que se resume aquí radica en que es uno de los pocos que intenta cuantificar en una misma métrica (los AVPP) el daño colateral producido por el cierre de las escuelas primarias, comparándolo con el que se habría producido si ésas hubiesen permanecido abiertas.

A nivel técnico el estudio es válido. Documenta adecuadamente las fuentes de datos utilizadas y es conservador en los valores que utiliza (no exagera para favorecer artificialmente la apertura de escuelas). También hace un análisis de sensibilidad para evaluar la robustez de los resultados. La mayor crítica que se le puede hacer está relacionada con las tasas de descuento social utilizadas $(0,0,5$ y $3 \%)$. Una tasa de descuento alta disminuye mucho los beneficios que se obtendrán en un futuro lejano (en este caso, menos AVPP en los niños que no pierden escolaridad), por eso argumentan que no debería usarse tasas altas en este caso. Pero muchos países sugieren tasas de descuento aún mayores a $3 \%$ (la máxima utilizada en el estudio, y donde los resultados entre dejar abiertas o cerradas las escuelas se equiparan bastante).

También es oportuno resaltar que este estudio es conservador porque no incluye el impacto en adolescentes por el cierre de escuelas secundarias, ni las disminuciones en la calidad de vida asociadas con las consecuencias de disminución de escolaridad de niños de hasta 11 años.

Como dato interesante, para el cálculo central de las consecuencias de la interrupción de la escolaridad utilizan un estudio sobre el impacto de las huelgas docentes en Argentina ${ }^{5}$. Si bien los resultados de los EE.UU. no son extrapolables a nuestro país, sí se puede argumentar que si se hiciera el mismo análisis aquí, los resultados podrían ser mucho peores, por la magnitud de la pérdida de días de clases presenciales ${ }^{6}$. Más aún, con los niveles de pobreza actuales, el cierre de las escuelas podría tener un impacto mucho más profundo en los alumnos argentinos, ya que en ellas no sólo reciben educación, 
sino un apoyo social mucho más amplio (incluyendo, para muchos, su comida más importante del día) ${ }^{7}$.

\section{Conclusiones del comentador}

Las decisiones sobre medidas tan dramáticas para combatir la pandemia no deberían ser políticas sino técnicas, y basadas en estudios como este. Además, es fundamental maximizar las probabilidades de que los niños accedan a la escuela, debido a sus terribles consecuencias, que se extienden por décadas. Pero abrir las escuelas no significa que se pueda prescindir de garantizar medios adecuados para minimizar el contagio en alumnos y trabajadores. Así como la pandemia exige una mejora integral y sostenida de las condiciones de trabajo del personal de la salud, también lo hace para el personal docente y no docente.

Gastón Perman [ Departamento de Salud Pública, Instituto Universitario Hospital Italiano de Buenos Aires; Área de Integración Socio-Sanitaria, Hospital Italiano de Buenos Aires; Servicio de Clínica Médica, Hospital Italiano de Buenos Aires. gaston.perman@hospitalitaliano.org.ar ]

Perman G Estimación de los años de vida potencial perdidos asociados al cierre de las escuelas primarias durante la pandemia de COVID-19. Evid Actual Pract Ambul. 2021;24(1):e002119. Comentado de: Christakis DA, et al. Estimation of US Children's Educational Attainment and Years of Life Lost Associated With Primary School Closures During the Coronavirus Disease 2019 Pandemic. JAMA Netw Open. 2020 Nov 2;3(11):e2028786. PMID: 33180132

\section{Referencias}

1. Christakis DA, Van-Cleve W, Zimmerman FJ. Estimation of US Children's Educational Attainment and Years of Life Lost Associated With Primary School Closures During the Coronavirus Disease 2019 Pandemic. JAMA Netw Open. 2020;3(11):e2028786. Available from: 10.1001/ jamanetworkopen.2020.28786.

2. Sánchez-R H, Albala-B C, Lera-M L. Años de vida perdidos por muerte prematura (AVPP) en adultos del Gran Santiago: ¿Hemos ganado con equidad? Rev méd Chile. 2005;133(5):575-582. Available from: 10.4067/s0034-98872005000500010;https://dx.doi.org/10.4067/s003498872005000500010 .

3. Castellano-Marrero D. Introducción a la estadística Bayesiana. Trabajo de Tesis de Grado de la Universidad Autónoma de Barcelona; 2015. Available from: https://ddd.uab.cat/pub/tfg/2015/137782/TFG_DailosCastellanoMarrero.pdf.

4. Perman G, Puga C, Ricci I, et al. Daños colaterales de la pandemia por COVID-19 ¿Consecuencias inevitables? Rev Hosp Ital Buenos Aires. 2020;40(4):185-90. Available from: https://www1.hospitalitaliano.org.ar/multimedia/archivos/noticias_attachs/47/documentos/115688_5-4020-Perman-A.pdf.

5. Jaume D, Willén A. The Long-Run Effects of Teacher Strikes: Evidence from Argentina. Journal of Labor Economics. 2019;37(4):1097-1139. Available from: 10.1086/703134;https://www.semanticscholar.org/paper/The-Long-Run-Effects-of-Teacher-Strikes\%3A-Evidence-Jaume-Will\%C3\%A9n/ b4b1362cb484dc8ddf5363429d5eff9eb8331bcc.

6. Análisis inicial de las NACIONES UNIDAS COVID-19 En Argentina: Impacto socioeconómico y ambiental; 2020. Available from: https://www. argentina.gob.ar/sites/default/files/informecovid19_argentina.pdf.

7. Grupo Banco Mundial Educación. COVID-19: Impacto en la educación y respuestas de política pública; 2020. Available from: https://openknowledge. worldbank.org/bitstream/handle/10986/33696/148198SP.pdf?sequence=6\&isAllowed $=y$. 